\title{
PDK1 promotes tumor cell proliferation and migration by enhancing the Warburg effect in non-small cell lung cancer
}

\author{
TAO LIU and HONGLEI YIN
}

\author{
Department of Pneumology, Heilongjiang Province Hospital, Harbin, Heilongjiang 150001, P.R. China
}

Received May 4, 2016; Accepted September 5, 2016

DOI: $10.3892 /$ or.2016.5253

\begin{abstract}
Tumor cells prefer glycolysis (Warburg effect) during the proliferation and metastasis. The precise mechanism remains largely unknown. Here, we demonstrated that pyruvate dehydrogenase kinase 1 (PDK1) was a critical enzyme that functioned as an oncogene to promote non-small cell lung cancer (NSCLC) growth and metastasis. We discovered that PDK1 expression was significantly upregulated in NSCLC tissues and correlated with advanced $\mathrm{T}$ stage. Moreover, high expression of PDK1 was an independent prognostic factor of NSCLC. Ectopic overexpression of PDK1 promoted cell proliferation and inhibited apoptosis. Also it was shown that PDK1 increased the cell mobility when Transwell assay was performed. Further experiments indicated that PDK1 had a central role in metabolic reprogramming by phosphorylating pyruvate dehydrogenase, leading to enhanced Warburg effect. Collectively, our data reveal a new function for PDK1, which could be used to indicate the prognosis of NSCLC, and provide targeted therapeutic strategy for clinical treatment.
\end{abstract}

\section{Introduction}

Lung cancer is one of the most common malignancies and results in large number of cancer-related deaths in the world. Approximately 1.6 million new cases are diagnosed annually. More than $85 \%$ of those cases are defined as non-small cell lung cancer (NSCLC) which is comprised of adenocarcinoma (ADC), squamous cell carcinoma (SCC), large-cell carcinoma (LLC) and other types (1). The diagnosis and clinical treatments of advanced NSCLC remains a great challenge of medical oncology and the 5-year survival rates is $<16 \%$, causing 1.4 million deaths annually (2). Hence, therapies aimed at understanding the disease development and progression, inhibiting further recurrences, or retarding

Correspondence to: Dr Honglei Yin, Department of Pneumology, Heilongjiang Province Hospital, 405 Gogol Street, Harbin, Heilongjiang 150001, P.R. China

E-mail: alexyee@sina.com

Key words: PDK1, Warburg effect, non-small cell lung cancer, proliferation, migration the tumor colony growth are urgently needed. Recently, the understanding of the molecular mechanisms of NSCLC has progressed significantly. Several oncogene amplifications and tumor-driven mutations have been identified such as KRAS, EGFR and ALK $(3,4)$. However, the biology of NSCLC still remains to be well characterized.

In cancer cells, aerobic glycolysis is one of the most important metabolic processes even if there is enough oxygen (5). This metabolic decision which divert pyruvate and its precursors undergo other anabolic processes or convert to lactate for cancer cells is known as the Warburg effect (6). Also the intermediates support nucleic, lipid and protein synthesis during the rapid cell division. So the Warburg effect supplies the high energy demands for cancer cell and biosynthesis materials. During these molecular adaptations, high pyruvate concentrations are maintained in cancer cells, which results in HIF-1 $\alpha$ stabilization (7). Then HIF- $1 \alpha$ acts as a key mediator that transcriptionally regulates expression of a vast variety of genes to promote cell proliferation, migration and metastasis (8-10). However, the molecular mechanism of increased glycolysis in NSCLC is largely unknown.

Pyruvate dehydrogenase (PDH) is a mitochondrial multienzyme complex that catalyzes the oxidative decarboxylation of pyruvate and is one of the major enzymes responsible for the regulation of homeostasis of carbohydrate $(11,12)$. Pyruvate dehydrogenase kinases (PDKs) could regulate PDH phosphorylation which leads to the inactivation of PDH (13). PDK1 has frequently increased expression in cancer cells including gastric cancer, colon cancer and acute myeloid leukemia (14-16). Besides, Koukourakis et al performed an immunohistochemical study and found that PDH/PDK pathway was repressed in $73 \%$ of NSCLC (17). However, the precise mechanisms and functions underlying the aberrant expression of PDK1 in NSCLC are still largely unclear, even controversy exist at present.

In this study, we used public database and our own clinical tissue samples to evaluate the expression and significance of PDK1 in NSCLC. PDK1 was closely associated with advanced $\mathrm{T}$ stage and multivariate analysis demonstrated that upregulated PDK1 was an independent prognostic factor. Moreover, ectopic expression or knockdown of PDK1 showed that PDK1 promoted cell proliferation and inhibited apoptosis via modulating the Warburg effect. These results provided new insights into the NSCLC progression. 


\section{Materials and methods}

Ethics and tissue specimens. Tissue microarray containing a total of 132 NSCLC cases was obtained from Outdo Biotech Co. (Shanghai, China). The age of these patients ranged from 20 to 84 (mean 60.5) and a 5-year follow-up was completed. The study was approved by the Ethics Committee of Heilongjiang Province Hospital.

Cell lines and transfection. NSCLC cell lines, including A549, H1299 and NCI-H460 were obtained form Cell Bank (SIBS, China). All cells were maintained in Dulbecco's modified Eagle's medium (DEME), supplemented with $10 \%$ fetal bovine serum and antibiotics $(100 \mathrm{U} / \mathrm{ml}$ penicillin and $100 \mu \mathrm{g} / \mathrm{ml}$ streptomycin). PDK1 overexpression plasmid pcDNA3.1-PDK1 and empty plasmid were transiently transfected into cells using Lipofectamine 2000 (Invitrogen, USA). Lentivirus containing PDK1 shRNA (RiboBio, China) were used to infect cells to establish the stable knock-down cell lines.

Immunohistochemical staining (IHC). The tissue microarray slides were deparaffinized, rehydrated in xylene and graded ethanol. Then antigens were retrieved at $95^{\circ} \mathrm{C}$ for $30 \mathrm{~min}$. After cooling to room temperature, the slides were incubated with 3\% hydrogen peroxide to block the endogenous peroxidase activity. Anti-PDK1 antibody was added to the slides overnight. After washing, the slides were incubated with HRP-labeled secondary antibody and stained using diaminobenzidine solution and counterstained with hematoxylin.

The IHC results were measured using a semi-quantitative score for both intensity and percentage of positive staining. Briefly, the intensity of staining was scored as: 1 (no stain), 2 (weak stain), 3 (medium stain), 4 (strong stain) and the percentage was scored as: $1(<25 \%), 2(25-50 \%), 3(50-75 \%)$, $4(75 \%)$. Then, the final score was calculated by multiplying the two scores and the overall score of 8 was the cut-off score which classified them into low expression and high expression groups.

Western blot analysis. Cell lysates were prepared using RIPA buffer with protease inhibitor and phosphatase inhibitor cocktail. Total protein $(70 \mu \mathrm{g})$ was loaded to $10 \%$ SDS-PAGE and transferred onto PVDF membranes. After blocking with $5 \% \mathrm{BSA}$, the membranes were blotted with primary antibody at $4^{\circ} \mathrm{C}$ overnight. Membranes were then incubated with peroxidase-conjugated secondary antibody at $37^{\circ} \mathrm{C}$. The blotting was visualized by enhanced chemiluminescence detection system. The antibodies used in this study were as followed: anti-PDK1 (10026-1-AP) from ProteinTech Group, Inc. (USA), anti-p-PDH (ab177461), anti- $\beta$-actin (ab8226) from Abcam (Cambridge, MA, USA) and anti-PDH (\#3205) from Cell Signaling Technology (MA, USA).

RNA extraction and quantitative real-time PCR (qRT-PCR). Total RNA was extracted by TRIzol reagent (Invitrogen) according to the manufacturer's insturctions. The first-strand cDNA was synthesized by PrimeScript RT Reagent kit (Takara, Japan). Quantitative real-time PCR was performed by SYBR Green method in ABI7500 system. The primers used in the study were as follows: PDK1, forward, 5'-CTCAGGACA
CCATCCGTTCA-3'; reverse, 5'-ACCATGTTCTTCTAGGC CTTTCAT-3'. $\beta$-actin, forward, 5'-TGATGATATCGCCGC GCTC-3'; reverse, 5'-CCATCACGCCCTGGTGC-3'. $\beta$-actin was used as internal control and the level of PDK1 was normalized using $2^{-\Delta \Delta c t}$ method.

Cell viability assay. For analyzing the viability of PDK1 overexpressing or knockdown cells, $2 \times 10^{3}$ cells/well were seeded in 96-well plates. Cell Counting Kit-8 (CCK-8, Dojindo, Japan) was used to measure the cell viability at $24,48,72$ and $96 \mathrm{~h}$ according to the manufacturer's insturctions. The absorbance was tested at $450 \mathrm{~nm}$. The experiments were repeated at least three times.

Colony formation assay. Indicated cells were seeded into the 6-well plate at 500 cells per well after 48 hours of transfected with vector, PDK1 plasmid or infected with PDK1 shRNA. The medium was changed every four days. After 2 weeks of incubation, colonies were fixed with 4\% paraformaldehyde and stained with crystal violet. The colony number was measured under a microscope. All of the experiments were performed at least three times.

Apoptosis assay. The apoptosis of cells with PDK1 ectopic expression or knockdown was analyzed using Annexin V/PI apoptosis detection kit (BD Bioscience, San Diego, CA, USA). Cells were trypsinized, collected in PBS, and resuspended in binding buffer. After incubation with Annexin V/PI for 30 min the proportion of Annexin V-positive cells were analyzed by FACSCalibur flow cytometer. All of the experiments were performed at least three times.

Migration assay. To detect cell migration ability, indicated cells $\left(10^{4}\right.$ in serum-free medium) were seeded into the top chamber of Transwell. The bottom chamber contained complete medium with $20 \%$ serum. After $48 \mathrm{~h}$ of incubation at $37^{\circ} \mathrm{C}$, cells on the lower membrane were fixed and stained with $0.1 \%$ crystal violet and imaged.

Lactate production, glucose uptake, oxygen consumption, ATP level detection. For lactate production measurement, cells were seeded into 96-well plates with phenol red-free medium and determined using Lactate assay kit (BioVision, CA, USA). For glucose uptake assay, the ${ }^{3} \mathrm{H}$-2-deoxyglucose was detected. Cells were cultured in 6-well plates and ${ }^{3} \mathrm{H}-2$-deoxyglucose was added to the cells and then washed with PBS and lysed in SDS. The radioactivity of cell lysates were detected. For oxygen consumption assay, the consumption rate was measured with MitoCell system (Strathkelvin Instruments, UK). ATP levels were measured using CellTiter-Glo Luminescent Viability Assay kit (Promega).

Statistical analysis. Statistical analysis was performed with SPSS 21.0 software (IBM, Chicago, IL, USA). The correlation between PDK1 expression and clinicopathological parameters was measured by Chi-square test. Survival rate was measured by Kaplan-Meier method and the significance was calculated with log-rank test. Univariate and multivariate analysis were performed by Cox regression model. Student's t-test was used to compare the groups in the cell experiments. 
Table I. Correlation between PDK1 expression and clinicopathological parameters of NSCLC.

\begin{tabular}{|c|c|c|c|c|}
\hline \multirow[b]{2}{*}{ Parameters } & \multicolumn{4}{|c|}{ PDK1 expression } \\
\hline & No. & Low & High & P-value \\
\hline Overall & 132 & 39 & 93 & \\
\hline \multicolumn{5}{|l|}{ Gender } \\
\hline Male & 69 & 17 & 52 & 0.196 \\
\hline Female & 63 & 22 & 41 & \\
\hline \multicolumn{5}{|l|}{ Age (years) } \\
\hline$<60$ & 60 & 10 & 42 & 0.917 \\
\hline$\geq 60$ & 72 & 21 & 51 & \\
\hline \multicolumn{5}{|c|}{ Tumor size (cm) } \\
\hline$<3$ & 37 & 11 & 26 & 0.977 \\
\hline$\geq 3$ & 95 & 28 & 67 & \\
\hline \multicolumn{5}{|c|}{ Tumor differentiation } \\
\hline Well & 20 & 6 & 14 & 0.998 \\
\hline Moderate & 75 & 22 & 53 & \\
\hline Poor & 37 & 11 & 26 & \\
\hline \multicolumn{5}{|l|}{ pT status } \\
\hline pT1 & 30 & 14 & 16 & $0.019^{\mathrm{a}}$ \\
\hline pT2-4 & 102 & 25 & 77 & \\
\hline \multicolumn{5}{|l|}{ pN status } \\
\hline $\mathrm{pN} 0$ & 85 & 27 & 58 & 0.452 \\
\hline pN1-N2 & 47 & 12 & 35 & \\
\hline
\end{tabular}

${ }^{\mathrm{a}} \mathrm{P}<0.05$.

Data are presented as the mean \pm SEM of three independent experiments. A P-value of $<0.05$ was considered statistically significant.

\section{Results}

PDK1 is upregulated in NSCLC tissues. To explore the relationship between the important enzymes involved in glycolysis and NSCLC progression, we analyzed the previous studies that identified numerous deregulated genes in NSCLC and focused on the glycolytic enzymes. We noted that PDK1 was upregulated in several studies which presented expression profiling by array. According to the GSE19188, containing a cohort of 91 tumor- and 65 adjacent normal lung tissue samples, PDK1 was significantly higher in tumor tissues compared to normal tissues (Fig. 1A). More importantly, the PDK1 expression in individual subtype of lung cancer, including adenocarcinoma (ADC), squamous cell carcinoma (SCC) and large cell carcinoma, was also enhanced (Fig. 1B). Furthermore, we employed another dataset to validate the hypothesis. As shown in Fig. 1C, the PDK1 expression in GSE10072 containing 180 cases of paired tumor samples was in conformity with our observation.

Then we examined the protein level of PDK1 in 132 pairs of NSCLC samples by immunohistochemical staining. The results revealed that PDK1 levels were significantly higher in tumor tissues than that in non-tumor tissues (Fig. 1D). We also analyzed the relationship between PDK1 and clinicopathological features. The results indicated that PDK1 expression was correlated with $\mathrm{T}$ stage $(\mathrm{P}=0.019)$ (Table I). These data suggested that PDK1 expression increased with the NSCLC development.

PDK1 is an independent prognostic factor of NSCLC. We explored the prognostic significance of PDK1 expression in NSCLC patients. All 132 NSCLC patients were divided into two groups: high expression (high PDK1, n=93) and low expression (low PDK1, n=38). Using Kaplan-Meier methods, we determined that patients with PDK1 high staining revealed significantly shorter survival rate compared with those with PDK1 low staining (Fig. 2A, $\mathrm{P}<0.0001$, log-rank test). We also performed univariate analysis to evaluate the associations between prognosis and clinicopathological features including age, gender, $\mathrm{T}$ stages, $\mathrm{N}$ stages, histological differentiation, tumor size and PDK1 expression. Among these features, advanced $\mathrm{T}$ stages $(\mathrm{P}=0.003)$, advanced $\mathrm{N}$ stages $(\mathrm{P}<0.0001)$ and $\mathrm{PDK} 1$-positivity $(\mathrm{P}=0.001)$ were significantly correlated with poor prognosis. Further multivariate regression analysis revealed that advanced $\mathrm{N}$ stages $(\mathrm{P}=0.001)$ and PDK1-positivity $(\mathrm{P}=0.001)$ were independent prognostic factors (Table II).

Table II. Univariate and multivariate analysis for ADC overall survival.

\begin{tabular}{|c|c|c|c|c|c|c|}
\hline \multirow[b]{2}{*}{ Parameters } & \multicolumn{3}{|c|}{ Univariate analysis } & \multicolumn{3}{|c|}{ Multivariate analysis $^{\mathrm{a}}$} \\
\hline & $\mathrm{RR}$ & $95 \% \mathrm{CI}$ & P-value & RR & $95 \% \mathrm{CI}$ & P-value \\
\hline Age & 1.147 & $0.721-1.825$ & 0.562 & - & - & - \\
\hline Gender & 1.108 & $0.699-1.755$ & 0.662 & - & - & - \\
\hline Histological differentiation & 1.166 & $0.837-1.625$ & 0.363 & - & - & - \\
\hline Tumor size & 1.306 & $0.767-2.224$ & 0.326 & - & - & - \\
\hline pT status & 3.080 & $1.475-6.432$ & 0.003 & 1.754 & $0.795-3.871$ & 0.164 \\
\hline pN status & 2.594 & $1.631-4.124$ & $<0.0001$ & 2.262 & $1.376-3.718$ & 0.001 \\
\hline PDK1 expression & 3.062 & $1.609-5.829$ & 0.001 & 2.879 & $1.500-5.527$ & 0.001 \\
\hline
\end{tabular}

${ }^{\text {a}}$ Final multivariate analysis includes only those covariates that were significantly associated with survival $(\mathrm{P}<0.05)$. 
A
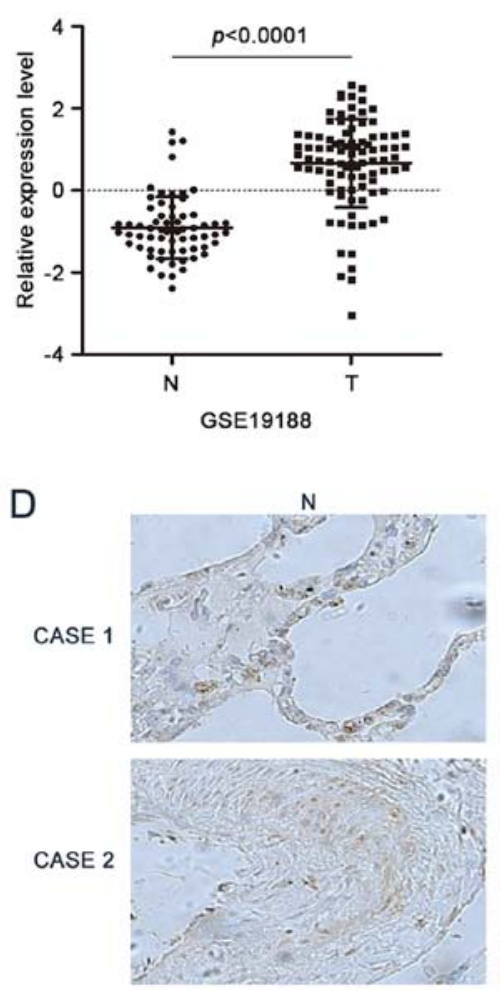

B

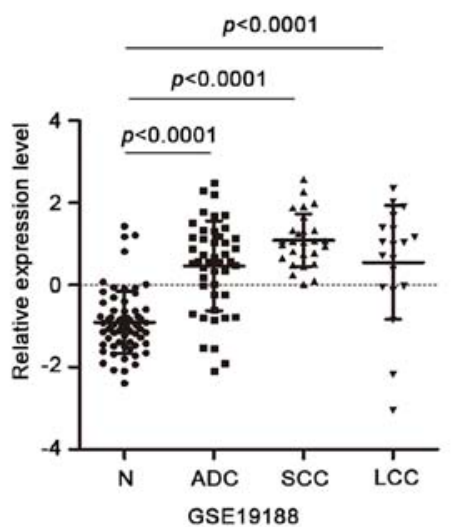

C

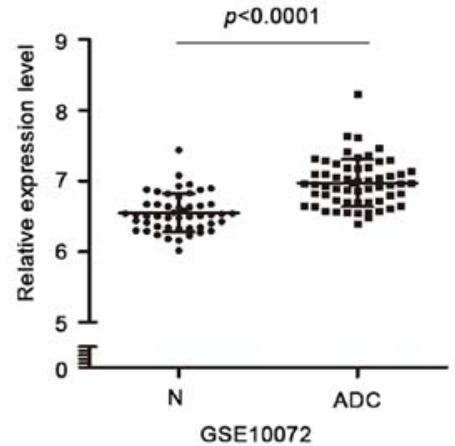

Figure 1. PDK1 is overexpressed in NSCLC tissues compared to normal tissues. (A) Relative PDK1 expression in DataSet GSE19188 (N, non-tumor tissues, T, tumors). (B) The expression of PDK1 in each subtype of NSCLC. (C) Relative PDK1 expression in DataSet GSE10072. (D) Representative images of the PDK1 expression in paired NSCLC tissues and non-tumor tissues (magnification, x200, left panel) and the statistical analysis of the IHC results (right panel).

A

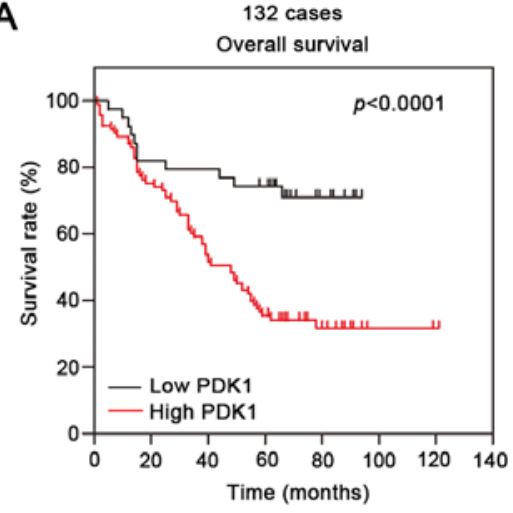

D

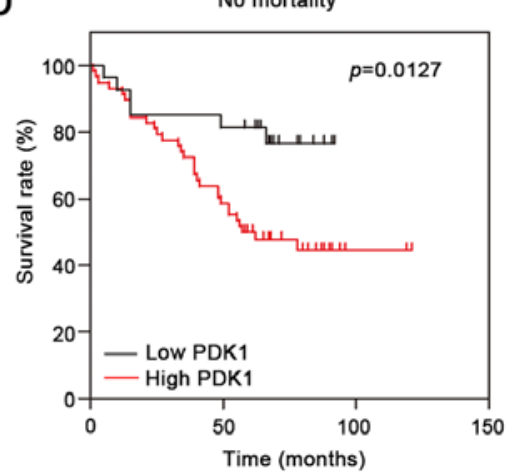

B

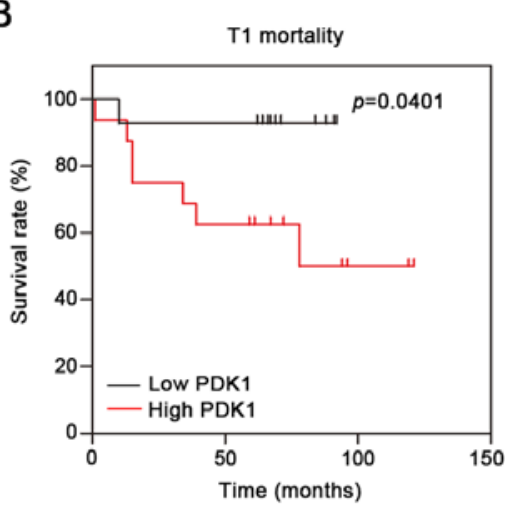

E

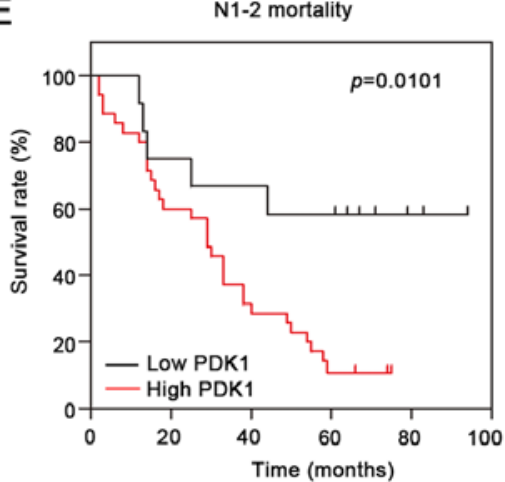

C

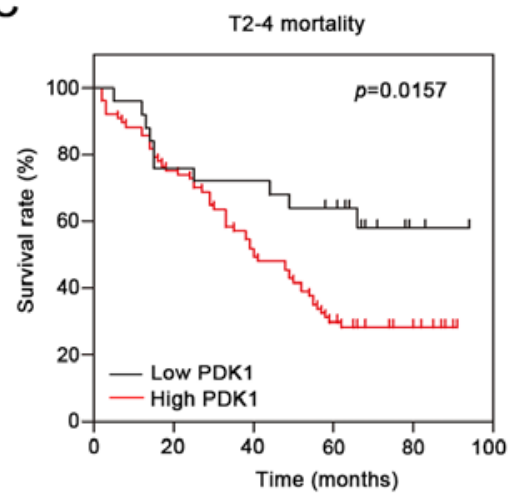

$\mathrm{F}$

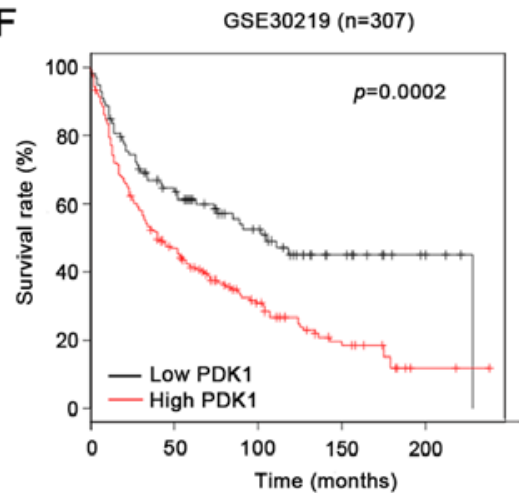

Figure 2. Kaplan-Meier analysis of PDK1 in NSCLC patients. (A) High and low expression of PDK1 were divided and survival in all NSCLC patients were analyzed. Log-rank regression was used to test the significance. Subsets of patients with T1 stages (B) and T2-4 stages (C). Subsets of patients with N0 stages (D) and N1-2 stages (E). (F) Kaplan-Meier analysis of NSCLC patients in DataSet GSE30219 according to PDK1 expression. 
A
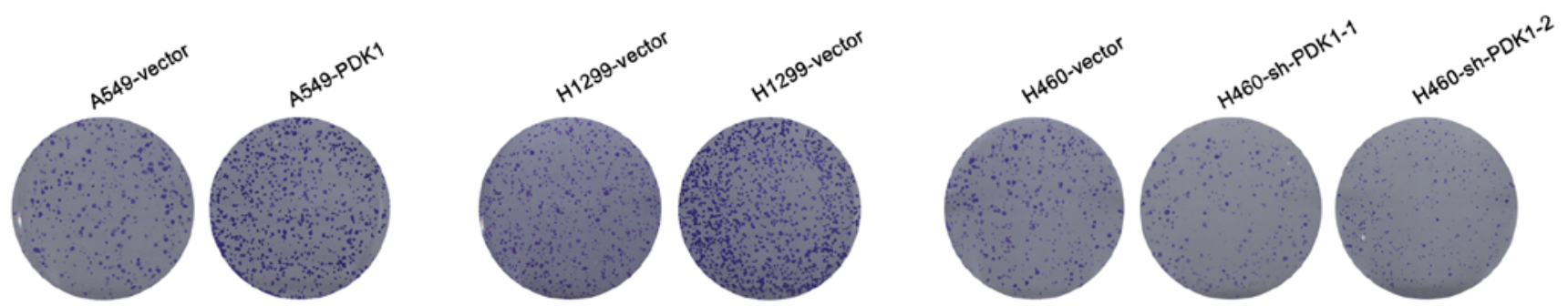

B
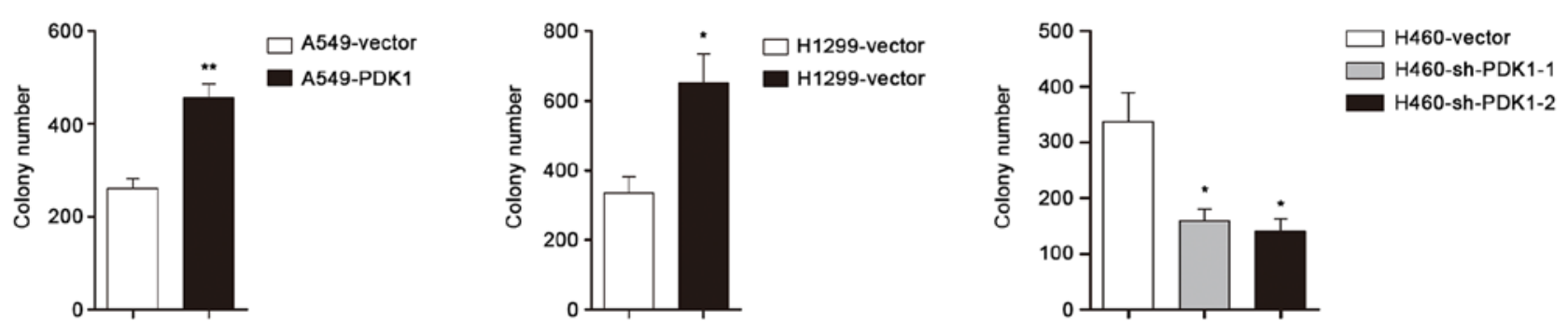

C
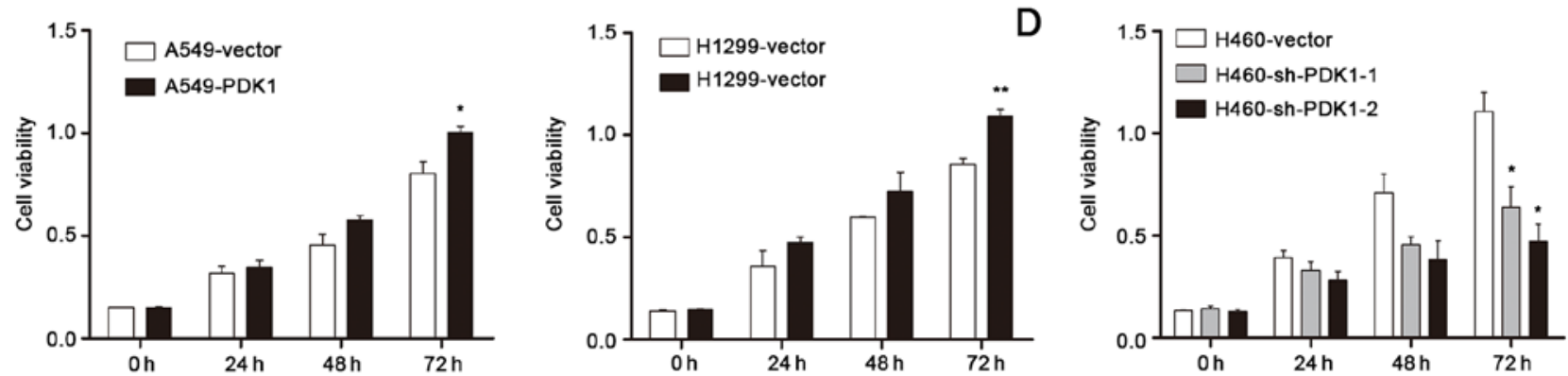

E

F
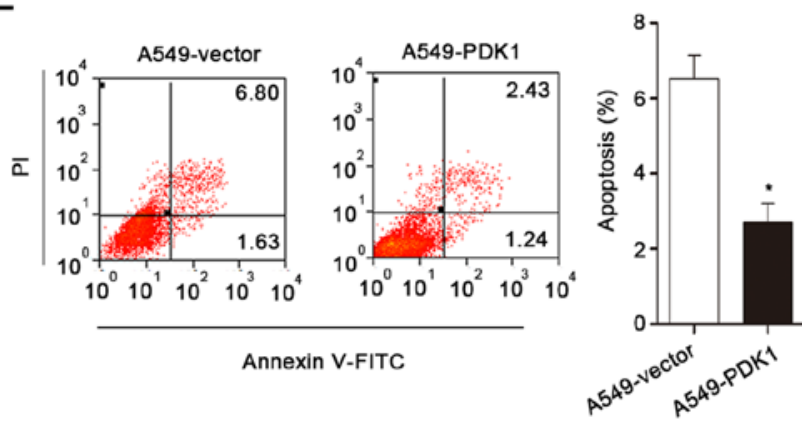

Annexin V-FITC
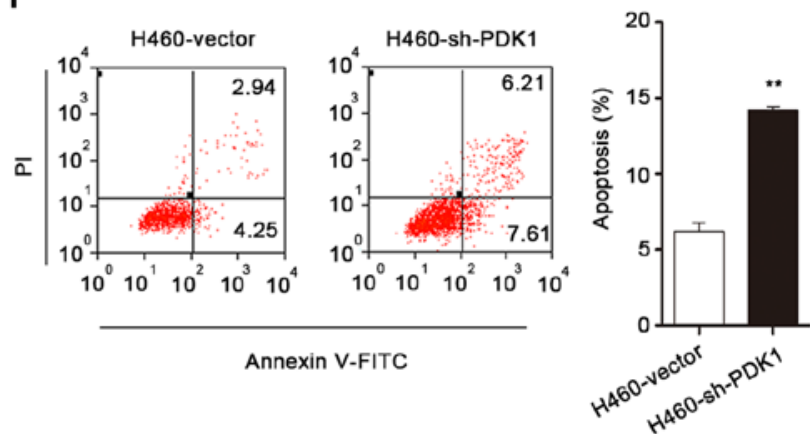

G
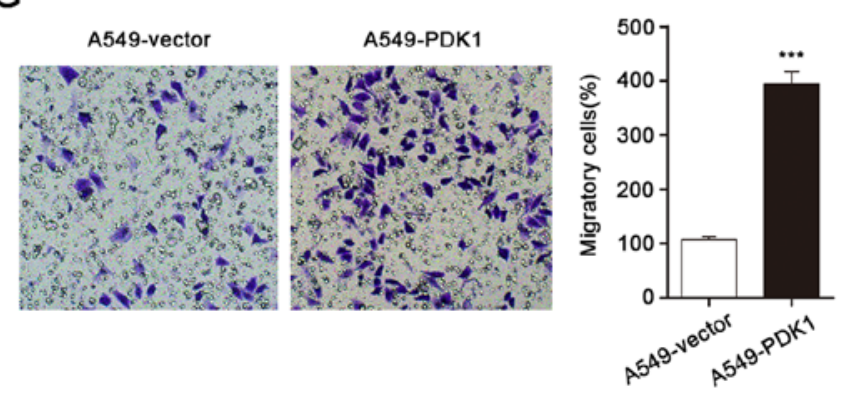

H

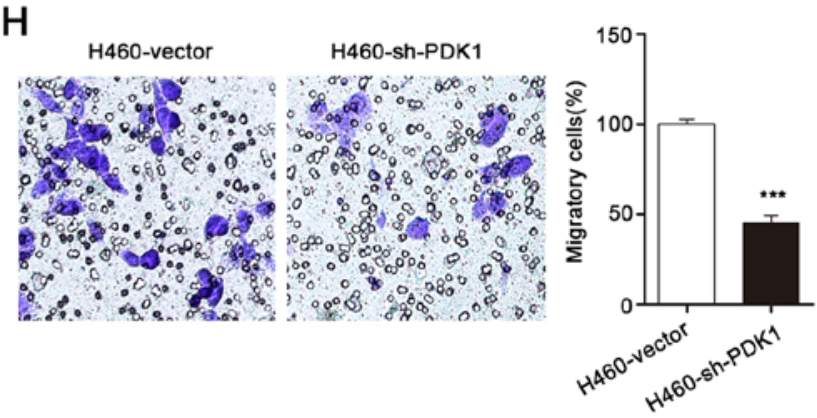

Figure 3. PDK1 promotes cell proliferation and migration of NSCLC cells. (A) Colony formation assay was used to measure the cell growth rate for ectopic expression and knockdown of PDK1. (B) Statistical analysis of colony formation assay of each group. The results were described as the relative number of five randomly selected fields. Cell viability of cells with PDK1 overexpression (C) and knockdown (D) were measured by CCK-8. Cell apoptosis rates with PDK1 overexpression (E) and knockdown (F) were measured by Annexin V/7-AAD. Representative images and statistical analysis of migration cells with PDK1 overexpression $(\mathrm{G})$ and knockdown $(\mathrm{H})$ (magnification, $\mathrm{x} 200)$. Data are presented as the mean \pm SEM of triplicate independent experiments $\left({ }^{*} \mathrm{P}<0.05,{ }^{* *} \mathrm{P}<0.01\right)$.

We then explored the correlations between PDK1 expression and prognosis using various subset analyzes. The results revealed that PDK1 was associated with shorter survival regardless of $\mathrm{T}$ stages or $\mathrm{N}$ stages (Fig. 2B-E). Moreover, we 
A

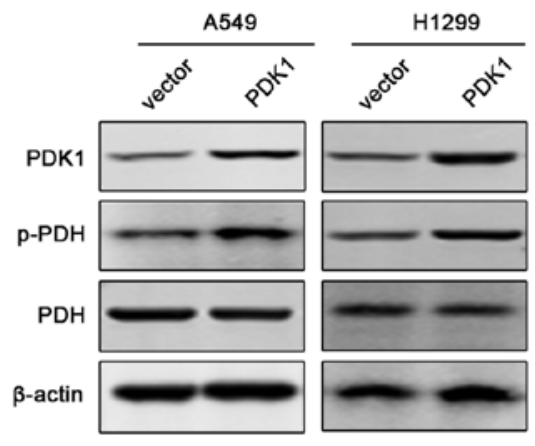

B

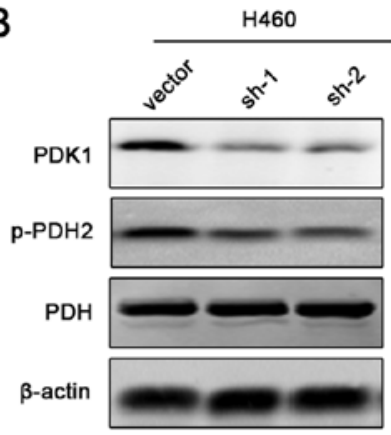

C

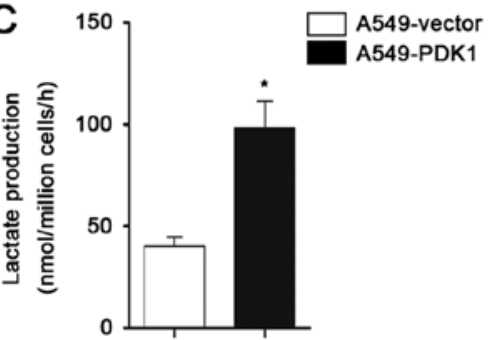

$E$

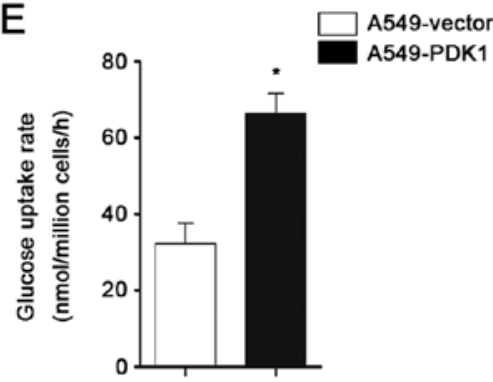

G

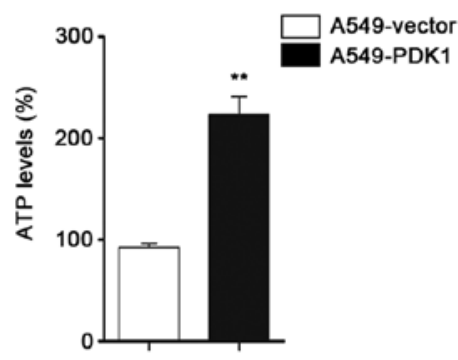

I

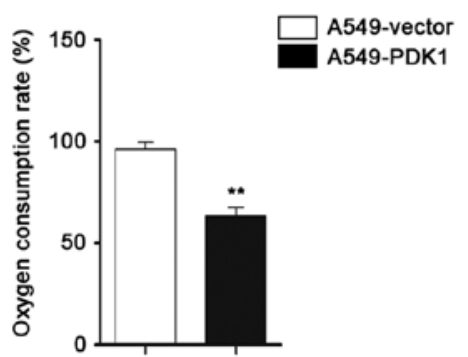

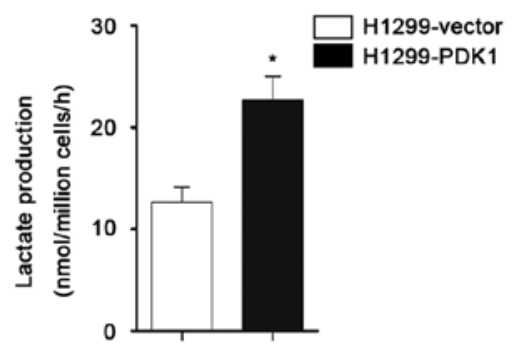
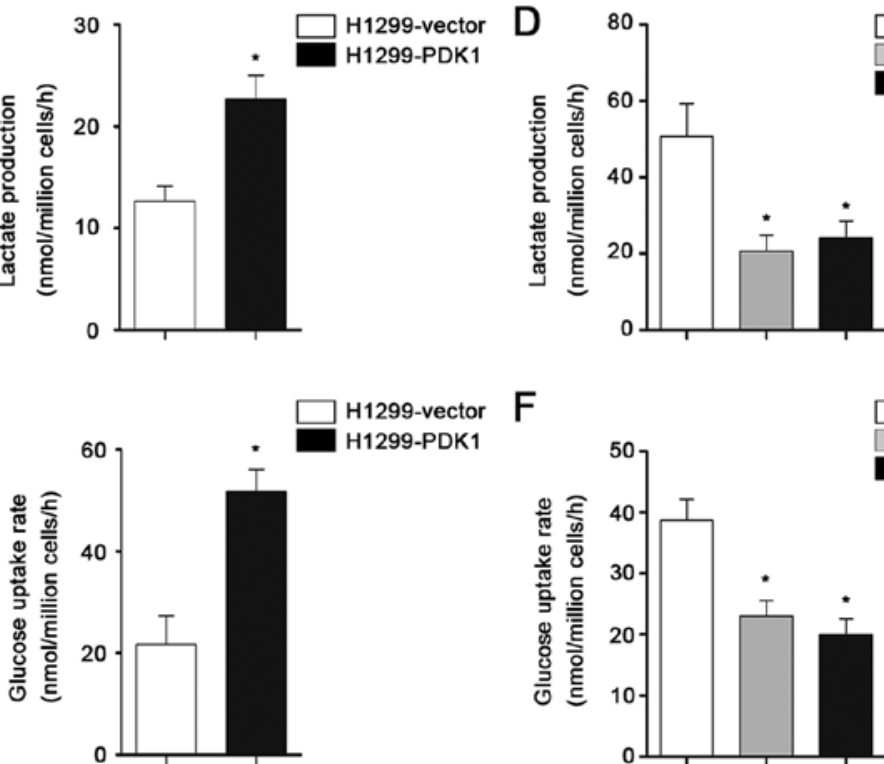

$F$
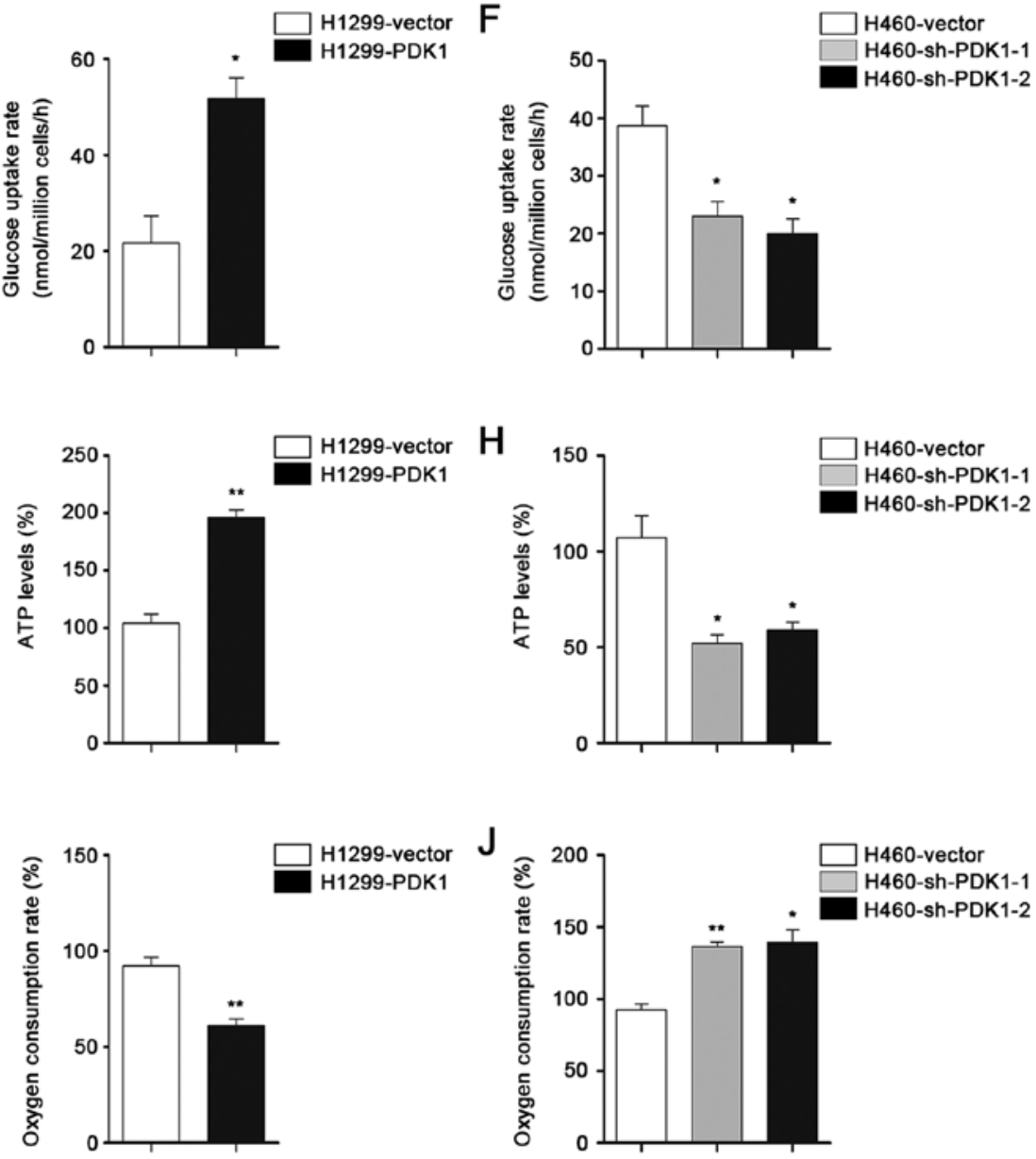

Figure 4. PDK1 modulates Warburg effects of NSCLC cells. Western blot analysis of cells with PDK1 overexpression (A) and knockdown (B). p-pyruvate dehydrogenase (p-PDH) and pyruvate dehydrogenase (PDH) were also detected. $\beta$-actin was used as internal control. Lactate production of cells with PDK1 overexpression (C) and knockdown (D). Glucose uptake of cells with PDK1 overexpression (E) and knockdown (F). ATP levels of cells with PDK1 overexpression $(\mathrm{G})$ and knockdown $(\mathrm{H})$. Oxygen consumption of cells with PDK1 overexpression (I) and knockdown (J). Data are presented as the mean \pm SEM of triplicate independent experiments $\left({ }^{*} \mathrm{P}<0.05,{ }^{* *} \mathrm{P}<0.01\right)$.

analyzed the PDK1 expression in GSE30219 and demonstrated that PDK1 could be a prognostic factor of NSCLC.
PDK1 promotes cell growth and inhibits apoptosis. For subsequent experiments, we performed ectopic expression of PDK1 
in A549 and H1299 cell lines and knockdown of PDK1 in H460 cells. The colony formation assay was carried out to evaluate the functions of PDK1 on proliferation of NSCLC cell lines. As shown in Fig. 3A and B, the statistical results indicated that overexpression of PDK1 could significantly promote cell growth while knockdown inhibited it. Moreover, the CCK- 8 assay was used to further determine the growth effect of PDK1. The results of CCK- 8 assay is shown in Fig. $3 \mathrm{C}$ and D, and were similar with colony formation assays. Also we performed FACS to explore whether the growth suppression was caused by apoptosis. Cell apoptosis was decreased after PDK1 overexpression (Fig. 3E). Conversely, knocking down of PDK1 markedly increased the percentage of Annexin V-positive cells (Fig. 3F). In addition, we employed Transwell assay to examine the effect of PDK1 on cell mobility (Fig. 3G and $\mathrm{H}$ ). The number of migratory cells with overexpression of PDK1 was increased significantly compared to control cells. Collectively, we suggested that PDK1 played oncogenic roles in NSCLC cells.

PDK1 modulates Warburg effect of NSCLC cells. As PDK1 is a key enzyme in modulating tumor cell glycolysis, we explored the function of PDK1 in regulating the Warburg effect. Western blot analysis was performed to measure the PDK1 overexpression or knockdown effects (Fig. 4A and B). Besides, p-PDH expression was increased by PDK1 phosphorylation. Measurement of metabolic parameters revealed that overexpression of PDK1 significantly increased cellular glucose uptake and lactate production (Fig. 4C and E), while knockdown of PDK1 decreased both glucose uptake and lactate production (Fig. 4D and F). Moreover, forced expression of PDK1 led to increased ATP levels and reduced $\mathrm{O}_{2}$ consumption (Fig. 4G and I). Contrary results were observed when we knocked down PDK1 (Fig. 4H and J). Collectively, we suggested that PDK1 could promote Warburg effect to facilitate cancer cell proliferation.

\section{Discussion}

Lung cancer is one of the most common malignant tumor which leads to over quarter of million deaths in America each year, and the 5-year survival is unfavorable. The heterogeneity of lung cancer is obvious, comprising adenocarcinoma, squamous cell carcinoma, large cell carcinoma and small cell carcinoma (18). Hence, the diagnosis and clinical treatments are much more difficult. NSCLC is a major subtype of lung cancer (19). Numerous studies have reported that metabolic reprogramming is a hallmark of many tumors, such as hepatocellular carcinoma, colorectal carcinoma, breast cancer and $\operatorname{NSCLC}(20,21)$. So it is meaningful and urgent to identify new markers and understand the mechanisms underlying the aberrant metabolism in cancer cells.

In this study, we analyzed the gene expression profiles in some published reports (22-24). Through microarray, we revealed that the expression of PDK1 was significantly upregulated in NSCLC tissues compared to normal tissues. Also, the immunohistochemical staining in protein level supported the mRNA results. In fact, PDK1 was reported to be overexpressed in various types of cancers including acute myeloid leukemia and colorectal carcinoma. Han et al reported that
miR-138, which could target PDK1, was found in sera and had prognostic potential (25). These results prompted us to further investigate PDK1 as an independent prognostic factor of NSCLC. Our study implied that high expression of PDK1 was associated with advanced T stage (75.5 vs $53.3 \%$ in early stage) and poor survival rate. Moreover, we determined the prognostic value of PDK1 by multivariate regression analysis. High expression of PDK1 was associated with poor prognosis whether in $\mathrm{T}$ stage or $\mathrm{N}$ stages.

PDK1 plays a critical role in regulating aerobic glycolysis by phosphorylating pyruvate dehydrogenase. Also, other substrates have been identified which were involved in tumor progression. In the case of AKT, PDK1 phosphorylated it by co-localizing in the plasma membrane (26). As for phosphorylation of S6K, SGK and RSK, the progress is dependent in conformational change $(27,28)$. As a consequence, the ability of PDK1 to induce multiple key molecules and signaling might participate in the development and progression of cancer. In this study, we observed that overexpression of PDK1 increased the cell proliferation rate and inhibited apoptosis while knockdown of PDK1 had the opposite effect. The impact might be due, at least partially, to enhanced Warburg effect as we detected.

Warburg effect ws recognized decades ago and has been paid attention to for a long time (29). The aberrant metabolic reprogramming could confer tumor cell growth advantage, drug resistance and metastasis not only by providing some raw molecules and precursors, but also regulating the reactive oxygen species (ROS) and tumor microenvironment $(30,31)$. Here, we detected the lactate production and glucose uptake with PDK1 overexpression or knockdown. In addition, PDK1 inhibited tricarboxylic acid cycle (TCA), however, as we observed, ATP level was increased with PDK1 overexpression. This could be due to the increased glucose uptake. So these results supported that PDK1 regulated aerobic glycolysis although there are still unknown factors that needed to be further investigated.

In conclusion, PDK1 was demonstrated to be overexpressed in NCSLS tissues and promoted cell proliferation and migration by modulating the Warburg effect. Importantly, high expression of PDK1 was significantly associated with advanced $\mathrm{T}$ stage and could act as an independent prognostic factor of NSCLC. Hence, further studies would be focused on the preferential inhibition of PDK1 as a strategy for NSCLC therapy.

\section{References}

1. Torre LA, Bray F, Siegel RL, Ferlay J, Lortet-Tieulent J and Jemal A: Global cancer statistics, 2012. CA Cancer J Clin 65: 87-108, 2015.

2. Reck M, Heigener DF, Mok T, Soria J-C and Rabe KF: Management of non-small-cell lung cancer: Recent developments. Lancet 382: 709-719, 2013.

3. Gainor JF, Varghese AM, Ou S-HI, Kabraji S, Awad MM, Katayama R, Pawlak A, Mino-Kenudson M, Yeap BY, Riely GJ, et al: ALK rearrangements are mutually exclusive with mutations in EGFR or KRAS: An analysis of 1,683 patients with non-small cell lung cancer. Clin Cancer Res 19: 4273-4281, 2013.

4. Gasparini P, Cascione L, Landi L, Carasi S, Lovat F, Tibaldi C, Alì G, D'Incecco A, Minuti G, Chella A, et al: microRNA classifiers are powerful diagnostic/prognostic tools in ALK-, EGFR-, and KRAS-driven lung cancers. Proc Natl Acad Sci USA 112: 14924-14929, 2015. 
5. Fong MY, Zhou W, Liu L, Alontaga AY, Chandra M, Ashby J, Chow A, O'Connor ST, Li S, Chin AR, et al: Breastcancer-secreted miR-122 reprograms glucose metabolism in premetastatic niche to promote metastasis. Nat Cell Biol 17: 183-194, 2015.

6. Yang W, Zheng Y, Xia Y, Ji H, Chen X, Guo F, Lyssiotis CA, Aldape K, Cantley LC and Lu Z: ERK1/2-dependent phosphorylation and nuclear translocation of PKM2 promotes the Warburg effect. Nat Cell Biol 14: 1295-1304, 2012.

7. Meijer TW, Kaanders JH, Span PN and Bussink J: Targeting hypoxia, HIF-1, and tumor glucose metabolism to improve radiotherapy efficacy. Clin Cancer Res 18: 5585-5594, 2012.

8. Gilkes DM, Bajpai S, Chaturvedi P, Wirtz D and Semenza GL: Hypoxia-inducible factor 1 (HIF-1) promotes extracellular matrix remodeling under hypoxic conditions by inducing P4HA1, P4HA2, and PLOD2 expression in fibroblasts. J Biol Chem 288: 10819-10829, 2013.

9. Li J, Xu Y, Long X-D, Wang W, Jiao HK, Mei Z, Yin QQ, Ma LN, Zhou AW, Wang LS, et al: Cbx4 governs HIF-1 $\alpha$ to potentiate angiogenesis of hepatocellular carcinoma by its SUMO E3 ligase activity. Cancer Cell 25: 118-131, 2014.

10. Pullamsetti SS, Banat GA, Schmall A, Szibor M, Pomagruk D, Hänze J, Kolosionek E, Wilhelm J, Braun T, Grimminger F, et al: Phosphodiesterase-4 promotes proliferation and angiogenesis of lung cancer by crosstalk with HIF. Oncogene 32: 1121-1134, 2013.

11. Kaplon J, Zheng L, Meissl K, Chaneton B, Selivanov VA, Mackay G, van der Burg SH, Verdegaal EM, Cascante M, Shlomi T, et al: A key role for mitochondrial gatekeeper pyruvate dehydrogenase in oncogene-induced senescence. Nature 498: 109-112, 2013.

12. Sutendra G, Kinnaird A, Dromparis P, Paulin R, Stenson TH, Haromy A, Hashimoto K, Zhang N, Flaim E and Michelakis ED: A nuclear pyruvate dehydrogenase complex is important for the generation of acetyl-CoA and histone acetylation. Cell 158: 84-97, 2014.

13. Sutendra G and Michelakis ED: Pyruvate dehydrogenase kinase as a novel therapeutic target in oncology. Front Oncol 3: 38, 2013.

14. Hur H, Xuan Y, Kim YB, Lee G, Shim W, Yun J, Ham IH and Han SU: Expression of pyruvate dehydrogenase kinase-1 in gastric cancer as a potential therapeutic target. Int J Oncol 42: 44-54, 2013.

15. Schell JC, Olson KA, Jiang L, Hawkins AJ, Van Vranken JG, Xie J, Egnatchik RA, Earl EG, DeBerardinis RJ and Rutter J: A role for the mitochondrial pyruvate carrier as a repressor of the Warburg effect and colon cancer cell growth. Mol Cell 56: 400-413, 2014

16. Qin L, Tian Y, Yu Z, Shi D, Wang J, Zhang C, Peng R, Chen X, Liu C, Chen Y, et al: Targeting PDK1 with dichloroacetophenone to inhibit acute myeloid leukemia (AML) cell growth. Oncotarget 7: 1395-1407, 2016.

17. Koukourakis MI, Giatromanolaki A, Sivridis E, Gatter KC and Harris AL; Tumor and Angiogenesis Research Group: Pyruvate dehydrogenase and pyruvate dehydrogenase kinase expression in non small cell lung cancer and tumor-associated stroma. Neoplasia 7: 1-6, 2005.
18. Li T, Kung H-J, Mack PC and Gandara DR: Genotyping and genomic profiling of non-small-cell lung cancer: Implications for current and future therapies. J Clin Oncol 31: 1039-1049, 2013.

19. Cooper WA, Lam DC, O'Toole SA and Minna JD: Molecular biology of lung cancer. J Thorac Dis 5 (Suppl 5): S479-S490, 2013.

20. Shimizu T, Inoue $K$, Hachiya $H$, Shibuya N, Shimoda $M$ and Kubota K: Frequent alteration of the protein synthesis of enzymes for glucose metabolism in hepatocellular carcinomas. J Gastroenterol 49: 1324-1332, 2014

21. Chiavarina B, Martinez-Outschoorn UE, Whitaker-Menezes D, Howell A, Tanowitz HB, Pestell RG, Sotgia F and Lisanti MP: Metabolic reprogramming and two-compartment tumor metabolism: Opposing role(s) of HIF $1 \alpha$ and HIF $2 \alpha$ in tumorassociated fibroblasts and human breast cancer cells. Cell Cycle 11: 3280-3289, 2012.

22. Hou J, Aerts J, den Hamer B, van Ijcken W, den Bakker M, Riegman P, van der Leest C, van der Spek P, Foekens JA, Hoogsteden HC, et al: Gene expression-based classification of non-small cell lung carcinomas and survival prediction. PLoS One 5: e10312, 2010.

23. Landi MT, Dracheva T, Rotunno M, Figueroa JD, Liu H, Dasgupta A, Mann FE, Fukuoka J, Hames M, Bergen AW, et al: Gene expression signature of cigarette smoking and its role in lung adenocarcinoma development and survival. PLoS One 3: e1651, 2008.

24. Rousseaux S, Debernardi A, Jacquiau B, Vitte A-L, Vesin A, Nagy-Mignotte H, Moro-Sibilot D, Brichon P-Y, Lantuejoul S, Hainaut $\mathrm{P}$, et al: Ectopic activation of germline and placental genes identifies aggressive metastasis-prone lung cancers. Sci Transl Med 5: 186ra166-186ra166, 2013.

25. Han L, Zhang G, Zhang N, Li H, Liu Y, Fu A and Zheng Y: Prognostic potential of microRNA-138 and its target mRNA PDK1 in sera for patients with non-small cell lung cancer. Med Oncol 31: 129, 2014

26. Martelli AM, Nyåkern M, Tabellini G, Bortul R, Tazzari PL, Evangelisti C and Cocco L: Phosphoinositide 3-kinase/Akt signaling pathway and its therapeutical implications for human acute myeloid leukemia. Leukemia 20: 911-928, 2006

27. Kang S, Dong S, Gu T-L, Guo A, Cohen MS, Lonial S, Khoury HJ, Fabbro D, Gilliland DG, Bergsagel PL, et al: FGFR3 activates RSK2 to mediate hematopoietic transformation through tyrosine phosphorylation of RSK2 and activation of the MEK/ERK pathway. Cancer Cell 12: 201-214, 2007.

28. Dann SG, Selvaraj A and Thomas G: mTOR Complex1-S6K1 signaling: At the crossroads of obesity, diabetes and cancer. Trends Mol Med 13: 252-259, 2007.

29. Vander Heiden MG, Cantley LC and Thompson CB Understanding the Warburg effect: The metabolic requirements of cell proliferation. Science 324: 1029-1033, 2009.

30. Upadhyay M, Samal J, Kandpal M, Singh OV and Vivekanandan P The Warburg effect: Insights from the past decade. Pharmacol Ther 137: 318-330, 2013

31. Yang F, Zhang H, Mei Y and Wu M: Reciprocal regulation of HIF-1 $\alpha$ and lincRNA-p21 modulates the Warburg effect. Mol Cell 53: 88-100, 2014. 